\title{
Valor nutricional de hortaliças folhosas não convencionais cultivadas no Bioma Cerrado
}

\section{Nutritional value of unconventional leafy vegetables grown in the Cerrado Biome/Brazil}

\author{
Neide Botrel ${ }^{1 *}$ (D), Sidinéia Freitas ${ }^{2}$, Marcos José de Oliveira Fonseca ${ }^{3}$, \\ Raphael Augusto de Castro e Melo ${ }^{4}$ (D), Nuno Madeira ${ }^{4}$
}

${ }^{1}$ Empresa Brasileira de Pesquisa Agropecuária (EMBRAPA), Centro Nacional de Pesquisas em Hortaliças, Laboratório de Ciência e Tecnologia de Alimentos, Brasília/DF - Brasil

${ }^{2}$ Empresa Brasileira de Pesquisa Agropecuária (EMBRAPA), Centro de Tecnologia Agrícola e Alimentar,

Departamento de Química de Alimentos, Rio de Janeiro/RJ - Brasil

${ }^{3}$ Empresa Brasileira de Pesquisa Agropecuária (EMBRAPA), Laboratório de Pós-colheita, Fisiologia e Tecnologia

Pós-colheita de Frutas e Hortaliças, Rio de Janeiro/RJ - Brasil

${ }^{4}$ Empresa Brasileira de Pesquisa Agropecuária (EMBRAPA), Centro Nacional de Pesquisas em Hortaliças,

Departamento de Fitotecnia, Brasília/DF - Brasil

${ }^{*}$ Corresponding Author: Neide Botrel, Empresa Brasileira de Pesquisa Agropecuária (EMBRAPA), Centro

Nacional de Pesquisas em Hortaliças, Laboratório de Ciência e Tecnologia de Alimentos, BR 060, km 09, Rodovia

Brasília-Anápolis, Fazenda Tamanduá Gama Oeste, CEP: 70359-970, Brasília/DF - Brasil, e-mail:

neidebotrel@me.com; nbotrel@yahoo.com.br

Cite as: Botrel, N., Freitas, S., Fonseca, M. J. O., Melo, R. A. C., \& Madeira, N. (2020). Nutritional value of unconventional leafy vegetables grown in the Cerrado Biome/Brazil. Brazilian Journal of Food Technology, 23, e2018174. https://doi.org/10.1590/1981-6723.17418

\begin{abstract}
Resumo
Hortaliças não convencionais ou tradicionais possuem uma vasta e rica variabilidade genética, sendo encontradas em feiras e outras formas de comercialização, em regiões nas quais fazem parte do contexto alimentar e cultural. Espécies de folhosas, como azedinha (Rumex acetosa), bertalha (Basella alba), capuchinha (Tropaeolum majus), ora-pro-nóbis (Pereskia aculeata), peixinho (Stachys byzantina), taioba (Xanthosoma taioba) e vinagreira (Hibiscus sabdariffa), entre outras, totalizando 14 espécies, foram cultivadas nas condições do Bioma Cerrado e caracterizadas quanto à sua composição centesimal e de minerais. Entre as hortaliças avaliadas, destaca-se o peixinho, com teor de fibra alimentar de $13,21 \mathrm{mg} 100 \mathrm{~g}^{-1}$. O teor de potássio em almeirão roxo, azedinha, beldroega e vinagreira

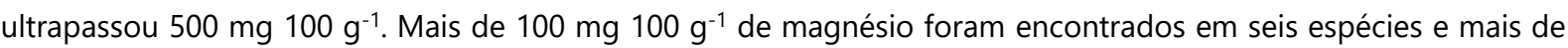

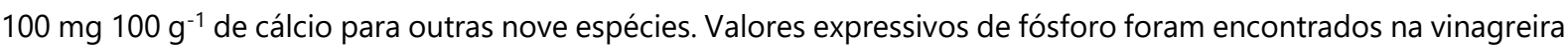

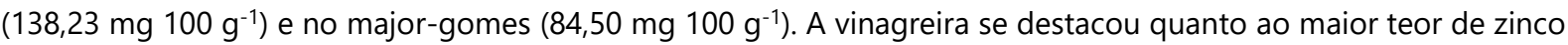

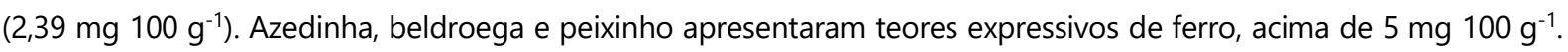
Esses valores são comparáveis ou superiores a hortaliças folhosas convencionais e denotam o potencial dessas hortaliças para consumo e comercialização, sendo necessária uma maior divulgação de seus valores nutricionais, devido aos altos teores de minerais encontrados somados à rusticidade dessas espécies, o que as tornam fontes alternativas de nutrientes disponíveis ao consumidor de hortaliças.
\end{abstract}

Palavras-chave: Composição de alimentos; Biodiversidade; Plantas espontâneas comestíveis; Nutrição; Hortaliças tradicionais; Minerais. 


\begin{abstract}
Traditional or unconventional vegetables have a wide and rich genetic variability; it is possible to found in farmers markets and other forms of commercialization, in regions where they are part of the food and cultural context. Species of leafy vegetables such as Rumex acetosa, Basella alba, Tropaeolum majus, Pereskia aculeata, Stachys byzantina, Xanthosoma taioba, Hibiscus sabdariffa, among others, totaling 14 species, were grown under the conditions of the Cerrado Biome/Brazil and characterized as to their proximate and mineral composition.

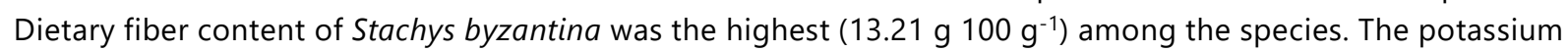
content in Lactuca canadenses, Rumex acetosa, Portulaca oleracea, and Hibiscus sabdariffa, exceeds $500 \mathrm{mg}$

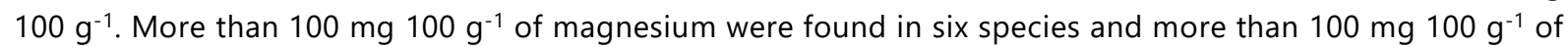
calcium for another nine species. Values of phosphorus in Talinum paniculatum and Hibiscus sabdariffa were $84.50 \mathrm{mg} 100 \mathrm{~g}^{-1}$ and $138.23 \mathrm{mg} 100 \mathrm{~g}^{-1}$, respectively. The Hibiscus sabdariffa (2.39 mg $100 \mathrm{~g} \mathrm{~g}^{-1}$ ) were prominent as good source of zinc. A Rumex acetosa, Portulaca oleracea e Stachys byzantina, plant presented expressive iron

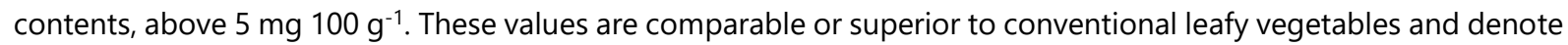
the potential of these species for consumption and commercialization, thus a greater dissemination of their nutritional values is necessary.
\end{abstract}

Keywords: Food composition; Biodiversity; Edible plants; Nutrition; Traditional vegetables; Minerals.

\title{
1 Introdução
}

Dentre as mais de 70 espécies olerícolas cultivadas no Brasil, existem aquelas pouco conhecidas pela maioria da população, que são as hortaliças não convencionais ou hortaliças tradicionais. São importantes em determinadas localidades por fazerem parte dos hábitos alimentares e da cultura regional (Madeira et al., 2013). Algumas hortaliças são de suma importância na expressão da cultura, como o ora-pro-nóbis, em algumas regiões de Minas Gerais; a vinagreira, no Maranhão, base do prato mais representativo da culinária desse estado - o arroz de cuxá; o jambu e a chicória-do-pará, no Norte do Brasil, entre outras. O cultivo e o consumo de hortaliças não convencionais têm diminuído em todas as regiões do país, em áreas rurais e urbanas, e entre todas as classes sociais, resultado da globalização e do crescente uso de alimentos industrializados, verificando-se mudanças significativas no padrão alimentar de brasileiros e perdas de características culturais e de identidade com o consumo de alimentos locais e regionais (Brasil, 2015).

De acordo com o Guia Alimentar para a População Brasileira (Brasil, 2014), os padrões tradicionais de alimentação, desenvolvidos e transmitidos ao longo de gerações, são fontes essenciais de conhecimentos para a formulação de recomendações que visam promover a alimentação adequada e saudável. Esses padrões resultam do acúmulo de conhecimentos sobre as variedades de plantas e de animais que mais bem se adaptaram às condições do clima e do solo, sobre as técnicas de produção que se mostraram mais produtivas e sustentáveis, e sobre as combinações de alimentos e preparações culinárias que bem atendiam à saúde e ao paladar humanos.

Além disso, algumas espécies correm risco de extinção em alguns estados, resultado do domínio da agricultura comercial de larga escala. Espécies de plantas espontâneas ou silvestres são, muitas vezes, consideradas "daninhas", "inços" ou "matos", fazendo com que suas utilidades e potencialidades econômicas sejam pouco conhecidas (Kinupp \& Lorenzi, 2014; Vieira et al., 2016). Tem-se como referência, no Brasil, o trabalho de Kinupp \& Lorenzi (2014), que trata das plantas alimentícias não convencionais (PANCs), com informações sobre várias espécies. Apresentam, entre outras vantagens, sua rusticidade e uma enorme diversidade de espécies. Porém, carecem de mais informações, destacando-se, no contexto deste trabalho, o seu valor nutricional, para um maior incentivo ao consumo. O objetivo deste estudo foi avaliar a composição centesimal e os teores de minerais em folhas de várias espécies de hortaliças não convencionais cultivadas no Bioma Cerrado. 


\section{Material e métodos}

As hortaliças folhosas não convencionais - anredera (Anredera cordifolia), azedinha (Rumex acetosa), almeirão-de-árvore (Lactuca canadenses), beldroega (Portulaca oleracea), bertalha (Basella alba), capuchinha (Tropaeolum majus), caruru (Amaranthus deflexus), jambu (Acmella oleracea), major-gomes (Talinum paniculatum), ora-pro-nóbis (Pereskia aculeata), peixinho (Stachys byzantina), serralha (Sonchus oleraceus), taioba (Xanthosoma taioba) e vinagreira (Hibiscus sabdariffa) - foram cultivadas na Embrapa Hortaliças, Brasília, Distrito Federal, localizada à altitude média de 996 metros e coordenadas geográficas de $15^{\circ} 56^{\prime} 00^{\prime \prime}$ de latitude Sul e $48^{\circ} 08^{\prime} 00^{\prime \prime}$ de longitude a Oeste. As amostras das folhas representativas de cada espécie foram colhidas pela manhã, selecionadas, descartando-se folhas apresentando danos físicos ou causados por doenças ou pragas, e em seguida enviadas ao Laboratório da Embrapa Agroindústria Alimentos, Rio de Janeiro-RJ, para realização das análises da composição centesimal e de minerais.

Foi realizada análise da composição centesimal, que trata de teor de proteína, umidade, cinzas, gordura (lipídeos) e carboidratos, dentre os quais a fibra alimentar. A análise da umidade foi realizada pelo método gravimétrico 931.0 (Association of Official Analytical Chemists, 2010). As amostras foram pesadas e levadas à estufa a $100{ }^{\circ} \mathrm{C}$ por aproximadamente $3 \mathrm{~h}$, até atingirem peso constante. $\mathrm{O}$ resíduo mineral fixo ou cinzas consistiu na destruição da matéria orgânica por queima em mufla a $550^{\circ} \mathrm{C}$ e pesagem do resíduo obtido, pelo método 923.03 (Association of Official Analytical Chemists, 2010).

$\mathrm{O}$ teor da proteína foi obtido através da determinação do nitrogênio total. $\mathrm{O}$ método utilizado foi o de Kjeldahl, baseado na digestão da amostra com ácido sulfúrico concentrado até que o carbono e o hidrogênio sejam oxidados. O nitrogênio da proteína foi reduzido e transformado em sulfato de amônia. Adicionou-se hidróxido de sódio e aqueceu-se para a liberação da amônia dentro de um volume conhecido de solução de ácido bórico. $\mathrm{O}$ borato de amônia formado foi titulado com ácido sulfúrico $0,05 \mathrm{M}$. $\mathrm{O}$ valor de nitrogênio obtido foi multiplicado por fator especifico da matriz para ser transformado no valor da respectiva proteína, pelo método 2001.11 (Association of Official Analytical Chemists, 2010). A gordura (lipídeos) foi determinada por equipamento automático, que realiza a extração da gordura da amostra por solvente - éter de petróleo (Association of Official Analytical Chemists, 2010).

A determinação de fibra alimentar total foi realizada em triplicata e acompanhada por dois ensaios em branco, pelo método enzimático-gravimétrico. A amostra desengordurada passou por três digestões. A primeira digestão foi feita em meio de tampão MES-TRIS ou tampão fosfato com enzima alfa-amilase em banho com agitação a $95{ }^{\circ} \mathrm{C}$ por 30 minutos. Em seguida, a amostra foi digerida com protease em $\mathrm{pH} 7,5$ a $60{ }^{\circ} \mathrm{C}$ por 30 minutos. Por último, a digestão foi feita em pH 4,5 com amiloglucosidase por 30 minutos a $60^{\circ} \mathrm{C}$. A solução resultante é adicionada de álcool etílico quente e, após uma hora, é filtrado em cadinho de Gooch. Todos os resíduos foram levados à estufa por $1 \mathrm{~h}$ a $100{ }^{\circ} \mathrm{C}$ e, após resfriamento, pesados. Cada um dos resíduos (branco e amostra) foi destinado à determinação de proteína e o outro, à determinação de cinzas. Estes valores foram descontados dos valores do resíduo obtido após a filtração, pelo método 985.29 (Association of Official Analytical Chemists, 2010). Para a determinação de minerais, as amostras foram pesadas em frasco apropriado e, em seguida, digeridas em micro-ondas, obedecendo a uma programação adequada. Após a digestão e o resfriamento dos frascos, as amostras foram avolumadas em frasco calibrado. Para a quantificação dos elementos presentes, as soluções foram colocadas em amostrador automático, para a leitura da concentração de cada elemento em ICP/OES. Os métodos utilizados foram para mineralização por micro-ondas de cavidade (Association of Official Analytical Chemists, 2010) e a quantificação por ICP-OES, pelo método 990 (Association of Official Analytical Chemists, 2010). Os teores de minerais foram expressos em base úmida. 


\section{Resultados e discussão}

Os valores obtidos da análise da composição centesimal das 14 espécies de hortaliças folhosas não convencionais encontram-se na Tabela 1, cujos dados das diversas características analisadas apresentaram relevantes variações, pois se trata, na maioria dos casos, de espécies diferentes. A umidade variou de 75,33\% a 93,53\%, no peixinho e no major-gomes, respectivamente. As espécies que apresentaram maior teor de umidade, superior a 91\%, são folhas suculentas, o que pode ser observado até mesmo de forma subjetiva, tais como nas espécies major-gomes, azedinha, beldroega e bertalha. Esses valores são índices importantes por refletirem os teores de umidade/matéria seca do produto, os quais podem interferir na estabilidade (reações químicas, bioquímicas e microbiológicas) e na textura, e consequentemente na sua vida útil. A fração de cinzas que representa as substâncias inorgânicas presentes no alimento apresentou uma variação de 1,05 a 4,21\%, na azedinha e no caruru, respectivamente (Tabela 1), indicando, de forma global, o conteúdo

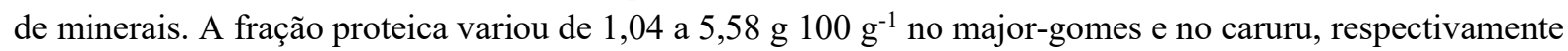
(Tabela 1). O teor proteico oferece uma informação de grande relevância do ponto de vista nutricional. Estudos mais específicos da qualidade da proteína, tais como seu valor biológico, grau de digestibilidade, quantidades de aminoácidos essenciais e de nitrogênio total, são essenciais (Blanco \& Bressani, 1991; Cozzolino, 1997). Algumas destas hortaliças já possuem estudos quanto a estes aspectos, como o ora-pro-nóbis, citado como uma hortaliça de boa digestibilidade (Takeiti et al., 2009). Os lipídios são moléculas altamente energéticas e, geralmente, aparecem em quantidades baixas em

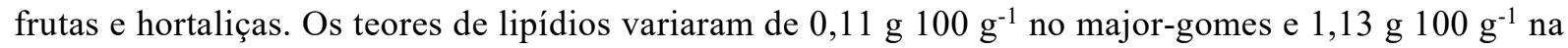
folha de capuchinha (Tabela 1). Os maiores teores de lipídeos são encontrados nas sementes, principalmente nas oleaginosas, nos tubérculos e em algumas frutas, valores que podem ultrapassar $60 \mathrm{~g} / 100 \mathrm{~g}$, como o caso da castanha-do-brasil (Somerville et al., 2000; Universidade Estadual de Campinas, 2011). As fibras apresentam benefícios à saúde, sendo as hortaliças uma boa opção para o suprimento diário deste constituinte. Das hortaliças analisadas, o peixinho apresentou um teor relevante de fibra alimentar de $13,21 \mathrm{~g}^{100 \mathrm{~g} \mathrm{~g}^{-1}}$

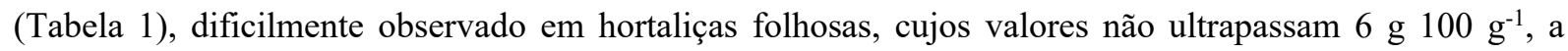

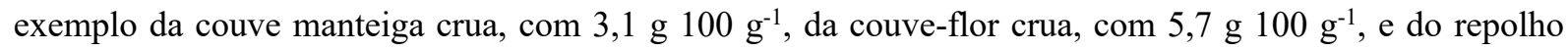

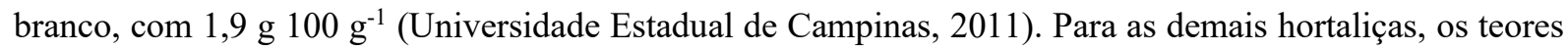

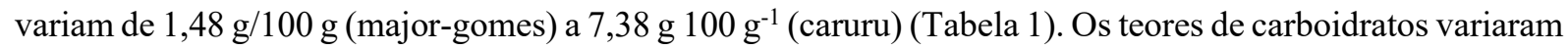

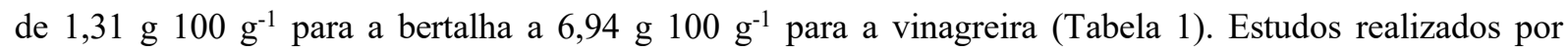
Ismail et al. (2008) relataram que as folhas de vinagreira Hibiscus sabdariffa contêm teores de proteína

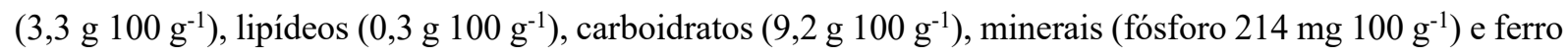
$\left(4,8 \mathrm{mg} 100 \mathrm{~g}^{-1}\right)$, corroborando os valores encontrados neste estudo.

Tabela 1. Composição centesimal, em base úmida, de folhas de 14 espécies de hortaliças não convencionais. Brasília-DF, 2017.

\begin{tabular}{cccccccc}
\hline \multirow{2}{*}{$\begin{array}{c}\text { Espécie } \\
\text { folhas) }\end{array}$} & Umidade $^{*}$ & Proteína $^{*}$ & Lipídeos $^{*}$ & Carboidrato $^{*}$ & $\begin{array}{c}\text { Fibra } \\
\text { alimentar }\end{array}$ & Cinzas $^{*}$ & \begin{tabular}{c} 
Valor $^{*}$ calórico $^{*}$ \\
\cline { 2 - 8 }
\end{tabular} \\
\hline Almeirão roxo & 90,25 & 1,73 & 0,53 & 2,73 & 3,44 & 1,33 & 22,57 \\
\hline Anredera & 89,6 & 1,36 & 0,67 & 4,01 & 2,47 & 1,45 & 27,57 \\
\hline Azedinha & 92,13 & 2,07 & 0,27 & 1,95 & 2,53 & 1,05 & 18,51 \\
\hline Bertalha & 93,25 & 2,01 & 0,21 & 1,31 & 2,02 & 1,2 & 15,17 \\
\hline Beldroega & 91,92 & 1,27 & 0,44 & 1,83 & 2,76 & 1,43 & 16,43 \\
\hline Capuchinha & 82,2 & 5,00 & 1,13 & 5,17 & 4,46 & 1,53 & 50,85 \\
\hline Caruru & 76,17 & 5,58 & 0,35 & 6,31 & 7,38 & 4,21 & 50,71 \\
\hline
\end{tabular}


Tabela 1. Continuação...

\begin{tabular}{cccccccc}
\hline \multirow{2}{*}{$\begin{array}{c}\text { Espécie } \\
\text { (folhas) }\end{array}$} & Umidade* $^{*}$ & Proteína $^{*}$ & Lipídeos $^{*}$ & Carboidrato $^{*}$ & $\begin{array}{c}\text { Fibra } \\
\text { alimentar }^{*}\end{array}$ & Cinzas $^{*}$ & $\begin{array}{c}\text { Valor } \\
\text { calórico }^{*}\end{array}$ \\
\cline { 2 - 9 } & & & $\left(\mathbf{g ~ 1 0 0 ~ \mathbf { g } ^ { - 1 } )}\right.$ \\
\hline Jambu & 82,09 & 3,85 & 0,26 & 4,37 & 6,53 & 2,90 & 35,22 \\
\hline Major-gomes & 93,53 & 1,04 & 0,11 & 2,28 & 1,48 & 1,57 & 14,23 \\
\hline Peixinho & 75,33 & 4,14 & 0,93 & 4,23 & 13,21 & 2,16 & 41,85 \\
\hline Ora-pro-nóbis & 88,65 & 2,1 & 0,51 & 2,65 & 3,88 & 2,33 & 22,62 \\
\hline Serralha & 87,32 & 3,57 & 0,52 & 2,42 & 4,34 & 1,84 & 28,6 \\
\hline Taioba & 86,58 & 3,05 & 0,62 & 4,12 & 3,89 & 1,74 & 34,26 \\
\hline Vinagreira & 81,52 & 3,97 & 0,63 & 6,94 & 5,3 & 1,53 & 49,31 \\
\hline
\end{tabular}

*Valores médios (três repetições) cada parâmetro analisado.

Os valores de quantificação de minerais das 14 espécies de hortaliças não convencionais foram expressos em base úmida. Dentre os macroelementos, foram analisados: sódio $(\mathrm{Na})$, potássio $(\mathrm{K})$, magnésio $(\mathrm{Mg})$, Cálcio $(\mathrm{Ca})$, manganês $(\mathrm{Mn})$ e fósforo $(\mathrm{P})$, os quais estão apresentados na Tabela 2. Algumas espécies apresentaram teores expressivos de distintos elementos, como o potássio no almeirão roxo, na azedinha, na beldroega, no major-gomes e na vinagreira, ultrapassando $400 \mathrm{mg} 100 \mathrm{~g}^{-1}$ (Tabela 2). Comparando-se os

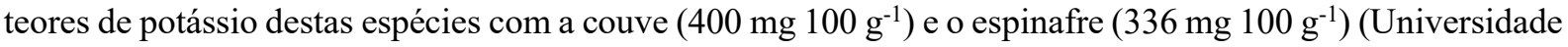
Estadual de Campinas, 2011), considerados boas fontes desse elemento, verifica-se que estas hortaliças podem ser uma excelente opção como fonte de potássio. Valores bastante elevados de sódio foram encontrados na serralha e na bertalha, de $26,33 \mathrm{mg} 100 \mathrm{~g}^{-1}$ e $8,21 \mathrm{mg} 100 \mathrm{~g}^{-1}$ (Tabela 2), respectivamente, porém estas também apresentaram altos teores de potássio, o que proporciona um balanço entre os dois elementos. Quanto aos teores de magnésio e cálcio, seis espécies apresentaram mais de $100 \mathrm{mg} 100 \mathrm{~g}^{-1} \mathrm{de}$ magnésio (anredera, azedinha, beldroega, bertalha, major-gomes e vinagreira) e nove espécies mais de $100 \mathrm{mg} 100 \mathrm{~g}^{-1}$ de cálcio (anredera, beldroega, bertalha, caruru, major-gomes, ora-pro-nóbis, peixinho, serralha e vinagreira) (Tabela 2). Comparando-se os resultados encontrados com teores citados para a couve

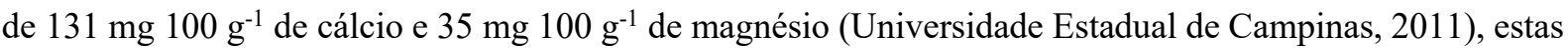
hortaliças podem ser uma boa opção para o suprimento de cálcio e magnésio na alimentação diária. $\mathrm{O}$ teor de cálcio encontrado para o major-gomes, em estudos realizados por Kinupp \& Barros (2008), também foi superior a $100 \mathrm{mg} 100 \mathrm{~g}^{-1}$. Os valores de cálcio mencionados na Tabela Brasileira de Composição de Alimentos - TACO (Universidade Estadual de Campinas, 2011) para as espécies serralha, taioba e caruru foram de 126,0 mg $100 \mathrm{~g} \mathrm{~g}^{-1} ; 141,0 \mathrm{mg} 100 \mathrm{~g} \mathrm{~g}^{-1}$, e 455,0 mg $100 \mathrm{~g}^{-1}$, respectivamente. De acordo com a Food and Agriculture Organization (2001) e Brasil (2003), a ingestão diária recomendada (IDR) de minerais pode variar conforme a idade da pessoa, entre outras condições, como lactente, mas, em média, as recomendações são: cálcio $1.000 \mathrm{mg}$; fósforo $(700 \mathrm{mg})$; potássio $(3.510 \mathrm{mg})$; magnésio $(260 \mathrm{mg})$; manganês $(2,3 \mathrm{mg})$; ferro $(14 \mathrm{mg})$; zinco $(7 \mathrm{mg})$, e cobre $(900 \mu \mathrm{g})$. Diferenças observadas nos valores obtidos neste trabalho, quando comparado com outros autores, podem ser influência de fatores pré-colheita, tais como local e sistema de cultivo, idade da planta no momento da colheita, bem como a variabilidade que pode ocorrer dentro da própria espécie. Cabe destacar a importância dos minerais para a saúde humana, os quais participam de várias reações químicas e bioquímicas no organismo, como o fósforo, que é um macroelemento de fundamental importância, cujas funções relacionam-se com a mineralização óssea e dos dentes, e que também participa do metabolismo energético, da absorção e no transporte de nutrientes, na regulação da atividade proteica e no balanço ácido-básico (Food and Agriculture Organization, 2001). Dentre as hortaliças não convencionais, ressaltam-se valores bastante expressivos de fósforo em várias espécies, em especial para o major-gomes $\left(84,50 \mathrm{mg} 100 \mathrm{~g}^{-1}\right) \mathrm{e} \mathrm{a}$

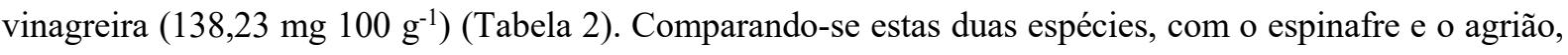


considerados fontes representativas de fósforo (25 mg $100 \mathrm{~g}^{-1}$ e $50 \mathrm{mg} 100 \mathrm{~g}^{-1}$, respectivamente) (Universidade Estadual de Campinas, 2011), verifica-se que estas hortaliças podem constituir uma boa fonte de fósforo.

Os microelementos, também conhecidos como elementos-traço, são considerados tão essenciais como as vitaminas. O zinco ( $\mathrm{Zn}$ ), por exemplo, é um elemento essencial ao organismo humano e, geralmente, as hortaliças folhosas são fontes modestas desse elemento (Food and Agriculture Organization, 2001). Neste

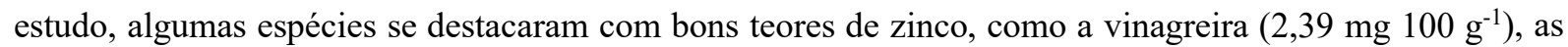
folhas de capuchinha $\left(0,76 \mathrm{mg} 100 \mathrm{~g}^{-1}\right)$ e a bertalha $\left(0,73 \mathrm{mg} 100 \mathrm{~g}^{-1}\right)$ (Tabela 2), sendo as duas últimas com teores similares ao citado para o agrião, que foi de $0,7 \mathrm{mg} 100 \mathrm{~g}^{-1}$ (Universidade Estadual de Campinas, 2011). A recomendação dietética de zinco para adultos é de $8 \mathrm{mg}$ por dia para mulheres e $11 \mathrm{mg}$ por dia para homens (Institute of Medicine, 2001). Dessa forma, estas hortaliças podem contribuir no suprimento diário desse microelemento. O manganês ( $\mathrm{Mn}$ ) no ora-pro-nóbis apresentou um teor bastante expressivo (7,31 mg $\left.100 \mathrm{~g}^{-1}\right)$, seguido do major-gomes (2,25 mg $\left.100 \mathrm{~g}^{-1}\right)$, em comparação com outras hortaliças, como alface, agrião, espinafre, repolho e rúcula, que não atingem $1 \mathrm{mg} 100 \mathrm{~g}^{-1}$ (Universidade Estadual de Campinas, 2011). Quanto ao Ferro (Fe), destacam-se as espécies azedinha, beldroega e peixinho, com teores superiores a $5 \mathrm{mg} 100 \mathrm{~g}^{-1}$ (Tabela 2), cujos teores ultrapassam teores de fontes tradicionais, como o espinafre $\left(0,4 \mathrm{mg} 100 \mathrm{~g}^{-1}\right)$ e a couve $\left(0,5 \mathrm{mg} 100 \mathrm{~g}^{-1}\right)$ (Universidade Estadual de Campinas, 2011). Os valores obtidos, também superiores ao citado por Oduro \& Owusu (2008), em Moringa oleifera, considerada como uma excelente fonte de ferro, cujo valor encontrado foi de 2,03 mg $100 \mathrm{~g}^{-1}$. Outro microelemento de grande importância para a saúde humana é o cobre, que, nas espécies bertalha, major-gomes, ora-pro-nóbis e vinagreira, apresentou teores em torno de $0,2 \mathrm{mg} 100 \mathrm{~g}^{-1}$. Comparando-se com os teores de cobre de outras hortaliças, como o espinafre, o repolho, o agrião e a chicória, citados na TACO (Universidade Estadual de Campinas, 2011), cujos teores variaram de $0,04 \mathrm{mg} 100 \mathrm{~g}^{-1}$ na chicória a $0,10 \mathrm{mg} 100 \mathrm{~g}^{-1}$ no agrião, as hortaliças não convencionais citadas superaram os teores de cobre dessas hortaliças.

Cabe destacar que os resultados apresentados neste trabalho demonstram que os teores de minerais são comparáveis ou superiores aos das hortaliças folhosas convencionais e denotam o potencial das hortaliças não convencionais para o consumo e comercialização, sendo necessária uma maior divulgação de seus valores nutricionais.

Tabela 2. Teores de minerais em base úmida de 14 espécies de hortaliças folhosas não convencionais. Brasília-DF, 2017.

\begin{tabular}{|c|c|c|c|c|c|c|c|c|c|}
\hline \multirow{2}{*}{ Espécie } & Sódio & Potássio & Magnésio & Cálcio & Manganês & Ferro & Zinco & Cobre & Fósforo \\
\hline & \multicolumn{9}{|c|}{$\left(\mathrm{mg} 100 \mathrm{~g}^{-1}\right)^{*}$} \\
\hline Anredera & 7,75 & 302,93 & 161,97 & 202,25 & 0,16 & 0,83 & 0,44 & 0,07 & 19,88 \\
\hline $\begin{array}{l}\text { Almeirão } \\
\text { roxo }\end{array}$ & 2,63 & 533,85 & 30,38 & 98,55 & 0,16 & 0,98 & 0,34 & 0,00 & 29,58 \\
\hline Azedinha & 2,77 & 623,31 & 105,03 & 84,40 & 0,93 & 5,87 & 0,45 & 0,14 & 46,00 \\
\hline Beldroega & 3,87 & 891,21 & 151,27 & 107,06 & 1,03 & 6,49 & 0,59 & 0,15 & 42,81 \\
\hline Bertalha & 8,21 & 304,34 & 165,75 & 186,59 & 1,06 & 2,88 & 0,73 & 0,20 & 53,87 \\
\hline Capuchinha & 1,88 & 167,74 & 34,15 & 73,21 & 0,27 & 0,46 & 0,76 & 0,08 & 43,63 \\
\hline Caruru & 4,55 & 304,47 & 66,11 & 139,72 & 0,17 & 2,07 & 0,39 & 0,04 & 21,45 \\
\hline Jambu & 1,64 & 230,14 & 27,83 & 69,36 & 0,57 & 3,80 & 0,14 & 0,07 & 19,87 \\
\hline Major gomes & 3,61 & 496,43 & 252,63 & 113,04 & 2,25 & 1,89 & 0,57 & 0,23 & 84,50 \\
\hline Ora-pro-nóbis & 5,42 & 322,98 & 94,46 & 269,38 & 7,31 & 1,33 & 0,28 & 0,25 & 17,61 \\
\hline
\end{tabular}


Valor nutricional de hortaliças folhosas não convencionais cultivadas no Bioma Cerrado

Botrel, N. et al.

Tabela 2. Continuação...

\begin{tabular}{|c|c|c|c|c|c|c|c|c|c|}
\hline \multirow{2}{*}{ Espécie } & Sódio & Potássio & Magnésio & Cálcio & Manganês & Ferro & Zinco & Cobre & Fósforo \\
\hline & \multicolumn{9}{|c|}{$\left(\mathrm{mg} 100 \mathrm{~g}^{-1}\right)^{*}$} \\
\hline Peixinho & $* *$ n.d. & 106,96 & 10,14 & 124,8 & 0,61 & 6,83 & 0,09 & 0,05 & 16,04 \\
\hline Serralha & 26,33 & 282,46 & 43,84 & 105,74 & 0,57 & 0,74 & 0,42 & 0,13 & 36,75 \\
\hline Taioba & 1,05 & 302,86 & 23,82 & 77,63 & 0,32 & 1,17 & 0,21 & 0,10 & 50,60 \\
\hline Vinagreira & 5,95 & 531,46 & 108,19 & 231,96 & 0,86 & 1,47 & 2,39 & 0,20 & 138,23 \\
\hline
\end{tabular}

*Valores médios (três repetições) de cada parâmetro analisado. **n.d. não detectável.

\section{Conclusão}

Conclui-se, com este estudo, o potencial nutricional das hortaliças não convencionais cultivadas no Bioma Cerrado. Os altos teores de minerais, somados à rusticidade dessas espécies, as tornam fontes alternativas de nutrientes disponíveis ao consumidor de hortaliças. No entanto, há necessidade de estudos complementares sobre a biodisponibilidade dos nutrientes.

\section{Referências}

Association of Official Analytical Chemists - AOAC. (2010). Official methods of analysis (18th ed., 1094 p.). Washington: AOAC. Blanco, A., \& Bressani, R. (1991). Biodisponibilidad de aminoácidos in el frijol (Plhaseolus vulgaris). Archivos Latinoamericanos de Nutricion, 41(1), 38-52. PMid:1822068.

Brasil. (2003, dezembro 26). Regulamento técnico sobre rotulagem nutricional de alimentos embalados (Resolução ANVISA/MS RDC n 360, de 23 de dezembro de 2003). Diário Oficial [da] República Federativa do Brasil, Brasília, seção 1.

Brasil. Ministério da Saúde. Secretaria de Atenção à Saúde. Departamento de Atenção. (2014). Guia alimentar para a população brasileira. (2. ed., 156 p.). Brasília: Ministério da Saúde.

Brasil. Ministério da Saúde. Secretaria de Atenção à Saúde. Departamento de Atenção. (2015). Alimentos regionais brasileiros (2. ed., 484 p.). Brasília: Ministério da Saúde.

Cozzolino, S. M. F. (1997). Biodisponibilidade de minerais. Revista de Nutrição, 10(2), 87-98. http://dx.doi.org/10.1590/S141552731997000200001

Food and Agriculture Organization - FAO. World Health Organization - WHO. (2001). Human vitamin and mineral requirements. In Food and Agriculture Organization - FAO. World Health Organization - WHO, Report 7th Joint FAO/OMS Expert Consultation (286 p.). Bangkok, Thailand: FAO/WHO.

Institute of Medicine. (2001). Food and nutrition board: Dietary reference intakes. Washington: National Academic Press.

Ismail, A., Ikram, E. H. K., \& Nazri, H. S. M. (2008). Roselle (Hibiscus sabdariffa L.) seeds nutritional composition protein quality and health benefits. Food, 2(1), 1-16.

Kinupp, V. F., \& Barros, I. B. I. (2008). Teores de proteína e minerais de espécies nativas, potenciais hortaliças e frutas. Food Science and Technology, 28(4), 846-857. http://dx.doi.org/10.1590/S0101-20612008000400013

Kinupp, V. F., \& Lorenzi, H. (2014). Plantas Alimentícias Não Convencionais (PANC) no Brasil: Guia de identificação, aspectos nutricionais e receitas ilustradas (768 p.). São Paulo: Instituto Plantarum de Estudos da Flora.

Madeira, R. N., Silva, P. C., Botrel, N., Mendonça, J. L., Silveira, G. S. R., \& Pedrosa, W. P. (2013). Manual de produção de hortaliças tradicionais (153 p.). Brasília: Embrapa.

Oduro, E. W., \& Owusu, D. (2008). Nutritional potencial of two leafy vegetables: Moringa oleífera and Ipomoe batatas leaves. Scientific Research and Essays, 3(2), 57-60.

Somerville, C. C., Browse, J., Jaworski, J. G., \& Ohlrogge, J. B. (2000). Lipids. In: B. Buchanan, W. Gruissem \& R. Jones (Eds.), Biochemistry \& molecular biology of plants. Rockville: American Society of Plant Phsyiologists. p.456-458.

Takeiti, C. Y., Antonio, G. C., Motta, E. M., Collares-Queiroz, F. P., \& Park, K. J. (2009). Nutritive evaluation of non-conventional leafy vegetable (Pereskia aculeata Miller). International Journal of Food Sciences and Nutrition, 60(1, Suppl. 1), 148-160. PMid:19468927. http://dx.doi.org/10.1080/09637480802534509

Universidade Estadual de Campinas - UNICAMP. Núcleo de Estudos e Pesquisas em Alimentação - NEPA. (2011). Tabela brasileira de composição de alimentos (TACO) (4. ed. rev. ampl., 161 p.). Campinas: NEPAUNICAMP. 
Vieira, R. F., Camillo, J., \& Coradin, L. (Eds.). (2016). Espécies nativas da flora brasileira de valor econômico atual ou potencial: Plantas para o futuro: Região Centro-Oeste (Série Biodiversidade, No. 44). Brasília: MMA.

Financiamento: EMBRAPA - Empresa Brasileira de Pesquisa Agropecuária - n. 03.13.01.004.00.00.

Received: Feb. 14, 2018; Accepted: Feb. 26, 2020 